\title{
Photochemical Behavior of Xanthophylls in the Recombinant Photosystem II Antenna Complex, CP26
}

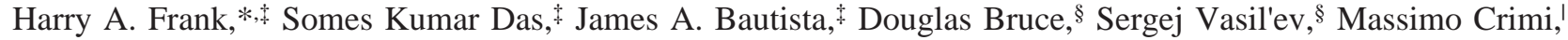 \\ Roberta Croce," and Roberto Bassi" \\ Department of Chemistry, University of Connecticut, Storrs, Connecticut 06269-3060, Department of Biological Sciences, \\ Brock University, St. Catherines, Ontario, Canada, and Facoltà di Scienze, Università di Verona, Verona, Italy
}

Received May 22, 2000; Revised Manuscript Received December 4, 2000

\begin{abstract}
The steady state absorption and fluorescence spectroscopic properties of the xanthophylls, violaxanthin, zeaxanthin, and lutein, and the efficiencies of singlet energy transfer from the individual xanthophylls to chlorophyll have been investigated in recombinant CP26 protein overexpressed in Escherichia coli and then refolded in vitro with purified pigments. Also, the effect of the different xanthophylls on the extents of static and dynamic quenching of chlorophyll fluorescence has been investigated. Absorption, fluorescence, and fluorescence excitation demonstrate that the efficiency of light harvesting from the xanthophylls to chlorophyll $a$ is relatively high and insensitive to the particular xanthophyll that is present. A small effect of the different xanthophylls is observed on the extent of quenching of $\mathrm{Chl}$ fluorescence. The data provide the precise wavelengths of the absorption and fluorescence features of the bound pigments in the highly congested spectral profiles from these light-harvesting complexes. This information is important in assessing the mechanisms by which higher plants dissipate excess energy in light-harvesting proteins.
\end{abstract}

Light-harvesting pigment-protein complexes in higher plants and algae possess a means of thermally dissipating photon energy absorbed, but not used, in photosynthesis. The process is known as nonphotochemical quenching (NPQ) ${ }^{1}$ and offers a short-term protective response to changes in incident light energy irradiance $(1-3)$. Dissipation of excess energy in light-harvesting proteins from photosynthetic systems can be measured quantitatively by observing the extent to which fluorescence from chlorophyll (Chl) $a$ bound in the complexes is affected by different chemical and physical conditions (4-11). NPQ is known to be associated with acidification of the thylakoid lumen and correlated with the enzymatic interconversion of violaxanthin and zeaxanthin in the xanthophyll cycle $(12,13)$. What is not clear, however, is precisely how the interconversion of the pigments in the xanthophyll cycle is associated with thermal dissipation. Two basic hypotheses for the involvement of the xanthophylls have been presented in the literature: direct quenching and indirect quenching.

The direct quenching model invokes specific energy states of xanthophyll molecules in the process of quenching

This work has been supported in the laboratory of H.A.F. by the National Science Foundation (Grant MCB-9816759), the National Institutes of Health (Grant GM-30353), and the University of Connecticut Research Foundation, and in the laboratory of R.B. by a MURST and CNR target project on Biotechnology.

* To whom correspondence should be addressed. Phone: (860) 486-

2844. Fax: (860) 486-6558. E-mail: harry.frank@uconn.edu.

$\doteqdot$ University of Connecticut.

$\S$ Brock University.

" Università di Verona.

${ }^{1}$ Abbreviations: Car, carotenoid; Chl, chlorophyll; CP, chlorophyll protein; LHC, light-harvesting complex; NPQ, nonphotochemical quenching; PS II, photosystem II; Xanth, xanthophyll.
$(1,13-15)$. Optical spectroscopic investigations of carotenoids and xanthophylls have shown that the energies of the lowest excited $\left(S_{1}\right)$ states of some of these molecules may be sufficiently low to quench $\mathrm{Chl}$ excited states, thus providing a means of regulating the flow of energy in the antenna (16-19). Determinations of the $\mathrm{S}_{1}$ excited state energies of violaxanthin and zeaxanthin suggest that although the direct route of quenching of excited states of Chl $a$ by these molecules is energetically feasible, the energy difference between the $S_{1}$ states of zeaxanthin and violaxanthin is not large enough to account for the magnitude of differential quenching associated with these pigments and observed in vivo $(20,21)$.

The indirect quenching model provides a role for the xanthophylls as modulators of the aggregation state of the pigment-protein complexes where regulation of the flow of energy in the antenna is achieved by the formation of quenching centers induced by xanthophyll binding to the light-harvesting assembly (4-8). The xanthophylls do not act as energy quenchers per se, but instead exert control over the aggregation state of the antenna complexes which leads to quenching.

The CP26 protein is one of a group of minor lightharvesting complexes from higher plants that is significantly enriched in the xanthophyll cycle pigments compared to the major LHC IIb protein (22-24). CP26 and the other minor light-harvesting complexes, CP24 and CP29, which are located close to the photosystem II (PS II) core, are between 210 and 257 amino acids long and bind five to six Chl $a$ and two to five $\mathrm{Chl} b$ molecules $(25,26)$, and their sequences ar between 29.2 and $48.7 \%$ homologous with LHC IIb (26). Because the CP26, CP24, and CP29 complexes bind the 
Table 1: Absorption Band Maxima $\left(\lambda_{\mathrm{ab}}{ }^{\max }\right)$, Fluorescence Band Maxima $\left(\lambda_{\mathrm{fl}}{ }^{\max }\right)$, Relative Quantum Yields $\left(\phi_{\mathrm{f}}\right)$, Percent Efficiencies $(\epsilon)$ of Energy Transfer from Xanth to $\mathrm{Chl} a$ and from $\mathrm{Chl} b$ to $\mathrm{Chl} a$, and Pigment Composition of the CP26 Complexes Reconstituted with the Various Xanthophylls ${ }^{a}$

\begin{tabular}{|c|c|c|c|c|c|c|c|c|c|c|c|}
\hline \multirow[b]{2}{*}{ CP26 sample } & \multirow[b]{2}{*}{$\lambda_{\mathrm{ab}}{ }^{\max }(\mathrm{nm})$} & \multirow[b]{2}{*}{$\lambda_{\mathrm{fl}}^{\max }(\mathrm{nm})$} & \multirow[b]{2}{*}{$\phi_{\mathrm{f}}(\%)$} & \multirow{2}{*}{$\begin{array}{c}\epsilon \text { for } \\
\text { Xanth } \rightarrow \text { Chl } a\end{array}$} & \multirow{2}{*}{$\begin{array}{c}\epsilon \text { for } \\
\mathrm{Chl} b \rightarrow \mathrm{Chl} a\end{array}$} & \multicolumn{6}{|c|}{ pigment composition } \\
\hline & & & & & & Chl $a$ & Chl $b$ & lutein & neox & viol & $\overline{\text { zeax }}$ \\
\hline control & $678 \pm 1$ & $682 \pm 1$ & $100 \pm 2$ & $97 \pm 3$ & $84 \pm 5$ & 6.1 & 2.9 & 1.0 & 0.55 & 0.45 & - \\
\hline violaxanthin & $677 \pm 1$ & $682 \pm 1$ & $106 \pm 3$ & $87 \pm 4$ & $96 \pm 4$ & 6.3 & 2.7 & - & - & 2.0 & - \\
\hline lutein & $677 \pm 1$ & $683 \pm 1$ & $100 \pm 2$ & $82 \pm 3$ & $80 \pm 5$ & 6.4 & 2.6 & 2.0 & - & - & - \\
\hline zeaxanthin & $676 \pm 1$ & $680 \pm 1$ & $62 \pm 2$ & $80 \pm 5$ & $43 \pm 5$ & 6.0 & 3.0 & - & - & - & 2.0 \\
\hline
\end{tabular}

${ }^{a}$ The efficiencies of energy transfer were obtained from a comparison of the absorption and excitation spectra of the CP26 complexes shown in Figure 3 as described in the text. The fluorescence quantum yields were measured relative to the control as described in the text.

majority of the xanthophyll cycle carotenoids, it has been suggested that they constitute the primary site of the NPQ mechanism and, as such, play an important role in regulating energy flow from the bulk LHC IIb to the PS II reaction center. Support for this assignment has emerged from the identification of protonation sites in CP26 and CP29 (2729 ), thought to correspond to the low luminal $\mathrm{pH}$ requirement for nonphotochemical quenching, and from the fact that CP29 is reversibly phosphorylated under stress conditions (30), suggesting an involvement in stress resistance.

Understanding precisely how the efficiency of lightharvesting or Chl fluorescence quenching correlates with the xanthophyll composition of the pigment-protein complexes has been hindered because natural complexes containing only one type of xanthophyll molecule cannot be isolated. In the work presented here, the spectroscopic properties, the efficiencies of singlet energy transfer, and the quenching of Chl fluorescence are examined in the isolated, unaggregated, CP26 light-harvesting complex containing only zeaxanthin, violaxanthin, or lutein. The studies were carried out on recombinant $\mathrm{CP} 26$ protein that was overexpressed in Escherichia coli and then refolded in vitro with purified $\mathrm{Chl}$ and xanthophyll pigments (31). Absorption, fluorescence, and fluorescence excitation spectroscopy reveal the spectral features and photochemical behavior that can be attributed to specific xanthophylls bound in the complexes. These experiments provide the precise wavelengths of the absorption and fluorescence features of the bound pigments in the typically congested spectral profiles from light-harvesting complexes from higher plants. This information is needed for assessing how these molecules contribute to the process of NPQ.

\section{MATERIALS AND METHODS}

Preparation of CP26 Complexes. CP26 apoprotein was overexpressed in E. coli strain SG13009. Purified inclusion bodies were reconstituted in vitro with pigments as previously described $(32,33)$. CP26 control protein was obtained by adding a total carotenoid mix, extracted from spinach thylakoids, to the reconstitution buffer together with the Chls. The CP26 samples containing the single xanthophyll species, lutein, violaxanthin, or zeaxanthin, were obtained using carotenoids purified by HPLC. The pigment analysis was performed by HPLC on a reversed phase $\mathrm{C}_{18}$ bondclone column detected with a Beckman system (Gold 126) equipped with a 168-element diode array (32). The Chl a/b ratio and carotenoid content were determined as described in ref 24, and the pigment/protein stoichiometry was determined from the fitting of the acetone extracts with the pure pigment absorption spectra. The products of reconstitution do not contain free pigments after the additional purification steps described in ref 32. All the recombinant proteins bound, within the experimental error, Chl $a$ and $\mathrm{Chl}$ $b$ in a mole ratio of $2 / 1$ and two xanthophyll molecules per polypeptide, suggesting that the carotenoid binding sites can bind each of the xanthophyll species. Control samples contained, on average, two molecules of lutein and either one molecule of violaxanthin or one molecule of neoxanthin per complex (see Table 1).

Sample Purification and Solvents. Chl $a$ and Chl $b$ were obtained from Sigma and were further purified by highperformance liquid chromatography (HPLC). Chl was dissolved in methanol and injected into a Millipore Waters 600E HPLC system using a Nova-Pak C18 column with mobile phases $\mathrm{A}$ (acetone), $\mathrm{B}$ (methanol), and $\mathrm{C}$ (water). The run was programmed as follows: from 0 to $10 \mathrm{~min}$, isocratic $58 \%$ acetone, $17 \%$ methanol, and $25 \%$ water; from 10 to 20 min, linear gradient to $60 \%$ acetone, $20 \%$ methanol, and $20 \%$ water; and from 20 to $50 \mathrm{~min}$, linear gradient to $75 \%$ acetone, $20 \%$ methanol, and $5 \%$ water. The eluent was monitored using the single-diode array detector, and the fractions containing Chls were collected and dried using a gentle stream of gaseous nitrogen. The purity was further confirmed by observing the similarity of the absorption spectra with the excitation spectra recorded at different emission wavelengths. The solvents were acetone (99.6\%), methanol $(99.9 \%)$, and distilled water from Fisher, and ethanol (100\%) from AAPER.

Spectroscopic Measurements. All of the spectroscopic experiments were carried out at room temperature unless otherwise noted.

Steady State Absorption and Fluorescence. Absorption spectra were recorded at room temperature using either a Milton Roy Spectronic 3000 Array photodiode array spectrometer or an Aminco DW200 spectrometer. Fluorescence spectroscopy was carried out at $12{ }^{\circ} \mathrm{C}$ using either an SLM Instruments, Inc., model 8000C spectrofluorimeter or a Jasco FP-777 spectrofluorimeter. For fluorescence excitation spectroscopy, the SLM Instruments spectrofluorimeter was used, and the spectral profile of the incident light was monitored using rhodamine 610 in ethylene glycol $(0.3 \mathrm{~g} / 100 \mathrm{~mL})$ as a quantum counter (34). The emission and incident light were detected by two independent Hamamatsu model R-928 photomultiplier tubes. The concentration of the samples was kept at $<10^{-6} \mathrm{M}$ which corresponds to an optical density at $677 \mathrm{~nm}$ of less than 0.01 in a $1 \mathrm{~cm}$ path.

Time-Resolved Fluorescence. Chlorophyll fluorescence decay kinetics were measured with the single-photon timing 


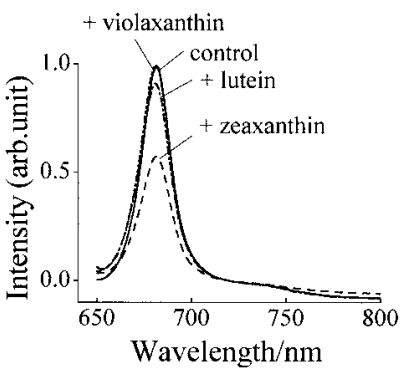

FIGURE 1: Fluorescence emission spectra of the recombinant CP26 complexes that have been reconstituted with $\mathrm{Chl} a$, Chl $b$, and different xanthophyll pigments. The emission spectra were taken using $630 \mathrm{~nm}$ excitation. The intensities of the bands were normalized to the value of the absorption of the sample at the excitation wavelength. The relative fluorescence quantum yields given in Table 1 were computed by integrating these spectra.

apparatus previously described (35). Excitation was at 664 $\mathrm{nm}$ with a repetition rate of $10 \mathrm{MHz}$. The fwhm of the system response function was approximately 80 ps. Fluorescence decays were recorded for several emission wavelengths at a resolution of $12 \mathrm{ps} / \mathrm{channel}$, resulting in a total time window of $12 \mathrm{~ns}$. The sample size was approximately $100 \mu \mathrm{L}$, and the samples were held in a $1 \mathrm{~mm}$ diameter capillary tube in a water jacket at $0{ }^{\circ} \mathrm{C}$. All of the samples were measured at the same total chlorophyll concentration. Fluorescence decay kinetics from all detection wavelengths were modeled globally with a sum of exponential decay components to produce decay-associated spectra.

Quantum Yields. The relative fluorescence quantum yields of the CP26 complexes were measured by integration of the area under the emission spectral profiles. This was carried out on samples that were first adjusted to an absorption value of 0.1 at $\sim 678 \mathrm{~nm}$ and then diluted by a factor of 200 . This provided samples of sufficient dilution that any effects of self-absorption were eliminated. This was checked further by an additional 4-fold dilution which showed no additional relative change. In each case, the amplitude of the emission signal was normalized on the basis of the absorption of the sample at the excitation wavelength of $630 \mathrm{~nm}$. The quantum yields are reported relative to a value of $100 \%$ assigned to the control sample. All spectral analyses, including addition, subtraction, multiplication, and integration, were carried out using Origin software (36).

\section{RESULTS AND DISCUSSION}

Steady State Spectroscopy. Figure 1 shows the emission spectra in the 600-800 $\mathrm{nm}$ region of the recombinant CP26 complexes that have been reconstituted with $\mathrm{Chl} a$, Chl $b$, and different xanthophyll pigments. Upon excitation at 630 $\mathrm{nm}$, the samples display a prominent peak at $682 \pm 1 \mathrm{~nm}$ corresponding to the maximum in the emission spectrum of $\mathrm{Chl} a$. A second smaller vibronic band in the emission profile occurs at $\sim 730 \mathrm{~nm}$ (Figure 1).

The fluorescence quantum yields of the $\mathrm{CP} 26$ complexes were measured by integrating the spectra shown in Figure 1. The values are given in Table 1 and represent the quantum yields of fluorescence relative to the control sample. The data show that incorporation of violaxanthin into the complex induces a small increase (by 6\%) in the relative fluorescence yield, while incorporation of zeaxanthin decreased the fluorescence yield to $62 \%$ of the control. Incorporation of
CP26+ zeaxanthin

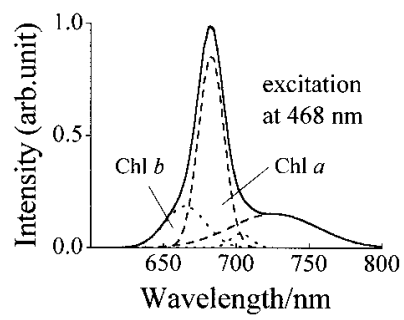

FIGURE 2: Gaussian deconvolution of the fluorescence emission spectrum generated using $468 \mathrm{~nm}$ excitation of the CP26 sample reconstituted with zeaxanthin. The spectrum was taken after freezing and thawing the sample which has the effect of dislodging some $\mathrm{Chl} b$ from the protein. The deconvolution of the spectrum reveals four vibronic components, two associated with Chl $a$ (dashed lines) and two associated with $\mathrm{Chl} b$ (dotted lines).

lutein showed no change in the integrated spectrum compared to the control.

The pigment composition of the control and the xanthophyll-reconsituted CP26 proteins is similar to that of the homologous CP29 protein. Work by Kuhlbrandt et al. (37) indicated that the xanthophylls on LHCIIb were bound to sites denoted L1 and L2, providing cross-bracing of helices A and B of the protein. In CP29, it was shown that site L1 binds lutein while site L2 binds either violaxanthin or neoxanthin with roughly equal probability (38). In LHCIIb, lutein in site L1 could be replaced with violaxanthin or zeaxanthin but not with neoxanthin, and furthermore, the occupancy of L1 was essential for protein folding (39). In CP29, the occupancy of site L1 was shown to be sufficient for protein stability (38). On this basis, it is proposed that the control CP26 sample binds lutein in site L1 while site L2 binds either neoxanthin or violaxanthin. When lutein is available during folding, together with other pigments, site L1 will be occupied by lutein, while the other xanthophyll species will be bound to site L2. It is therefore the occupancy of site L2 that modulates the fluorescence yield of CP26: Lutein is neutral with respect to the control sample, violaxanthin increases fluorescence emission, and zeaxanthin induces quenching. The control sample is, in fact, a mixed population of two kinds of CP26 molecules differing in the occupancy of site L2 with either violaxanthin or neoxanthin.

All of the samples were stable to repetitive freezing and thawing with the exception of the sample containing zeaxanthin. If the CP26 sample reconstituted with zeaxanthin was frozen and thawed, the emission spectrum developed a noticeable shoulder at $\sim 660 \mathrm{~nm}$ (see Figure 2). In this sample, excitation at $630 \mathrm{~nm}$ produced an emission spectrum with a maximum at $680 \pm 1 \mathrm{~nm}$, which is shifted slightly to the blue compared to those of the other samples. A blue shift of the same magnitude is also observed in the red-most absorption band from this preparation (data not shown). This suggests that a small difference in the pigment-pitment or pigment - protein interactions is induced by the incorporation of zeaxanthin into the protein structure. Gaussian deconvolution of the emission spectrum from this sample revealed that the shoulder at $\sim 660 \mathrm{~nm}$ is associated with $\mathrm{Chl} b$ emission. Chl $b$ emission in antenna complexes from higher plants is not usually detected in steady state fluorescence experiments because of rapid and efficient transfer of energy from $\mathrm{Chl} b$ to $\mathrm{Chl} a$. The presence of $\mathrm{Chl} b$ emission in the CP26 sample incorporating zeaxanthin probably occurred due 
to a fraction of the Chl $b$ normally bound in the protein becoming dislodged from its native binding site. This "free" Chl $b$ would be expected to be more fluorescent than the Chl $b$ bound in its native environment which can transfer energy to Chl $a$. The efficiency of energy transfer from the dislodged Chl $b$ to Chl $a$ would be expected to be greatly reduced or eliminated altogether compared to the efficiency of energy transfer between the bound $\mathrm{Chl} b$ and $\mathrm{Chl} a$. The fact that this observation was restricted to the sample containing zeaxanthin and that this sample displayed spectral bands shifted compared to those of the other complexes indicate that this complex is less stable to protein folding into the native CP26 configuration than the complexes which incorporate violaxanthin or lutein.

Panels $\mathrm{A}-\mathrm{H}$ of Figure 3 show the absorption and excitation spectra in the region of the Chl $a$ and $b$ Soret and xanthophyll absorption bands from 350 to $550 \mathrm{~nm}$. The solid lines correspond to the absorption (panels A, C, E, and G) and excitation (panels B, D, F, and H) spectra of the CP26 complexes reconstituted with the different xanthophylls. The combination dashed and dotted lines correspond to the absorption and excitation spectra of purified Chl $a$ in ethanol. The dotted lines correspond to the absorption and excitation spectra in the Soret region of purified Chl $b$ in ethanol. The spectra of Chl $a$ and $\mathrm{Chl} b$ in ethanol were shifted between 4 and $7 \mathrm{~nm}$ to account for the small effect of environment on the Chl peak positions and to align the spectra better with the absorption maxima of the CP26 complexes. The dashed lines represent the absorption spectra of the various xanthophylls (Xanth) taken in ethanol and shifted by between 18 and $22 \mathrm{~nm}$ to accommodate the large spectral shift associated with the change in polarizability of the medium $(40,41)$. No changes in the overall line shapes of the spectra of the xanthophylls were required to simulate the absorption or excitation profiles of the CP26 complexes because it has been demonstrated that for xanthophylls lacking carbonyl functional groups, no effect on the width or vibronic structure is associated with changes in solvent environment (41). For the control sample, the xanthophyll line shape was constructed from a sum of the absorption spectra of lutein, neoxanthin, and violaxanthin in a 2/1/1 molar ratio consistent with the xanthophyll composition of the reconstituted CP26 complex (Table 1). The stoichiometric ratios of $\mathrm{Chl} a$ to $\mathrm{Chl}$ $b$ deduced from the simulations of the absorption spectra shown in Figure 3 were approximately $2 / 1$ for all the complexes. This is consistent with the stoichiometry of the native CP26 complex $(25,26)$ and the pigment composition determined by HPLC analysis (Table 1).

The integrated areas of the xanthophyll bands (dashed lines in Figure 3) in the absorption and excitation spectra were used to calculate the efficiencies of energy transfer between the xanthophyll and $\mathrm{Chl} a$ and $b$. Because the emission from the CP26 complexes at $\sim 680 \mathrm{~nm}$ (Figure 1) derives essentially entirely from $\mathrm{Chl} a$, the absorption and excitation spectra shown in Figure 3 were normalized to the maximum intensities of the Chl $a$ spectra in its Soret region at 435 $\mathrm{nm}$. Comparing the integrated areas of the absorption and excitation line shapes associated with the xanthophylls for the control CP26 sample (Figure 3G,H) revealed an overall efficiency of energy transfer between the xanthophylls and Chl $a$ of $97 \pm 3 \%$ and an efficiency of energy transfer between $\mathrm{Chl} b$ and $\mathrm{Chl} a$ of $84 \pm 5 \%$. A similar analysis for

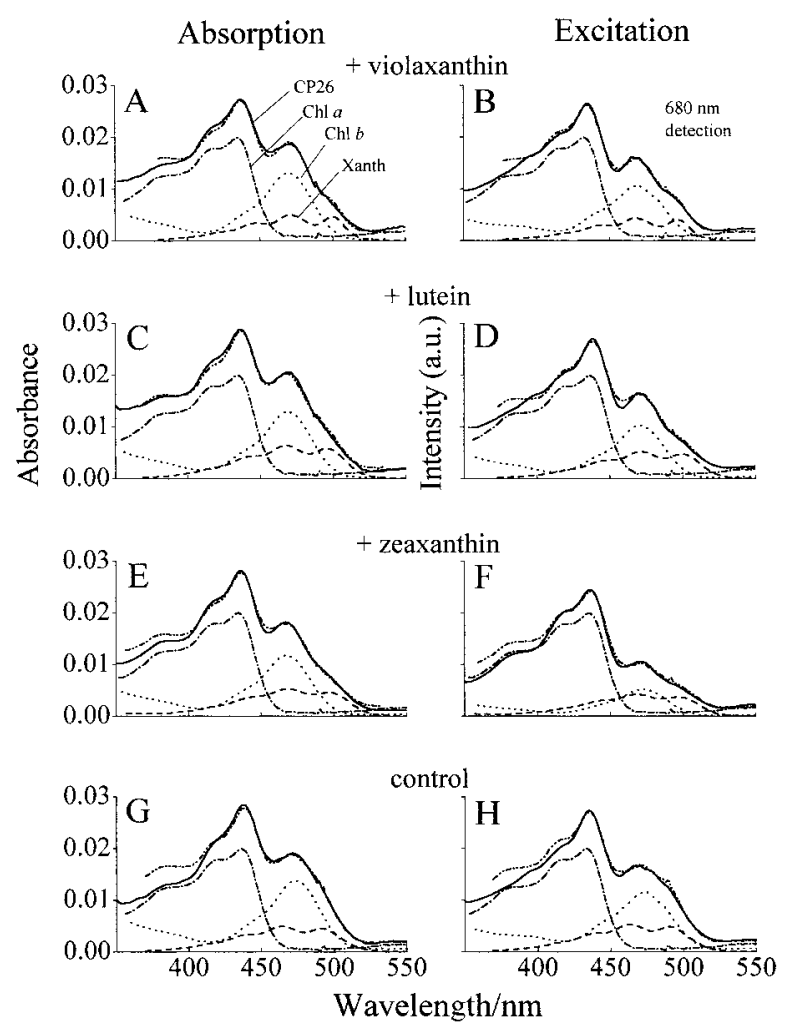

FIGURE 3: Absorption and excitation spectra in the Chl $a$ and Chl $b$ Soret and xanthophyll absorption region from 350 to $550 \mathrm{~nm}$. The solid lines correspond to the experimental absorption (A, C, $\mathrm{E}$, and $\mathrm{G}$ ) and excitation (B, D, F, and $\mathrm{H}$ ) spectra of the CP26 complexes reconstituted with the different xanthophylls. The combination dashed and dotted lines correspond to the absorption and excitation spectra of purified Chl $a$ in ethanol. The dotted lines correspond to the absorption and excitation spectra in the Soret region of purified $\mathrm{Chl} b$ in ethanol. The spectra of $\mathrm{Chl} a$ and Chl $b$ in ethanol were shifted between 4 and $7 \mathrm{~nm}$ to account for the small effect of environment on the Chl peak positions and to align the spectra better with the absorption maxima of the CP26 complexes. The dashed lines represent the absorption spectra of the various xanthophylls (Xanth) taken in ethanol and shifted between 18 and $22 \mathrm{~nm}$ to accommodate the large spectral shift associated with the change in the polarizability of the medium. The dashed and double-dotted lines are simulations of the experimental line shapes generated by summing the $\mathrm{Chl} a, \mathrm{Chl} b$, and xanthophyll spectra shown in the figure.

the CP26 sample containing violaxanthin (Figure 3A,B) revealed an efficiency of energy transfer between the xanthophyll and Chl $a$ of $87 \pm 4 \%$ and an efficiency of energy transfer between $\mathrm{Chl} b$ and $\mathrm{Chl} a$ of $96 \pm 4 \%$. For the CP26 sample containing lutein (Figure 3C,D), the efficiencies were measured to be $82 \pm 3 \%$ between the xanthophyll and Chl $a$ and $80 \pm 5 \%$ between $\mathrm{Chl} b$ and $\mathrm{Chl}$ $a$. These high efficiencies can be seen qualitatively in the fact that the ratio of the intensity of the xanthophyll excitation band to the Chl $a$ excitation band (panel B, D, or $\mathrm{H}$ of Figure 3 ) is similar to the ratio of the intensity of the xanthophyll absorption band to the Chl $a$ absorption band (panel A, C, or $\mathrm{G}$ of Figure 3).

A comparison of the absorption and fluorescence excitation spectra from the CP26 sample reconstituted with zeaxanthin revealed an efficiency of energy transfer between the xanthophyll and Chl $a$ of $80 \pm 5 \%$. Also, the sample containing zeaxanthin had an efficiency of $43 \pm 5 \%$ for energy transfer between $\mathrm{Chl} b$ and $\mathrm{Chl} a$. This is significantly lower than the efficiency observed for the other complexes 


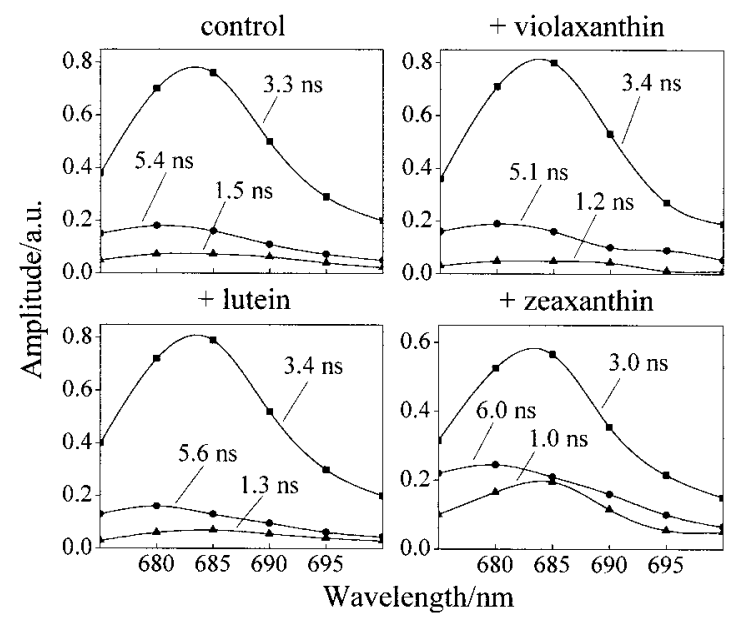

FIGURE 4: Decay-associated fluorescence spectra of the CP26 complexes taken using $664 \mathrm{~nm}$ excitation as described in the text. All of the samples were measured at the same total chlorophyll concentration. Fluorescence decay kinetics from all detection wavelengths were modeled globally with a sum of exponential decay components to produce decay-associated spectra. The spectra associated with the three kinetic components used to fit the fluorescence transient data are shown.

and very likely due to the fact that, as discussed above, because of the instability of this complex, some $\mathrm{Chl} b$ has been dislodged from the protein. The difference in behavior is evident from the fact that the $\mathrm{Chl} b$ contribution to the excitation spectrum of the CP26 sample containing zeaxanthin (dotted line in Figure 3F) is much smaller than the $\mathrm{Chl}$ $b$ contribution to the absorption spectrum (dotted line in Figure 3E). Also, the excitation spectrum from this sample was dependent on the wavelength of detection (data not shown). If the excitation spectrum was taken with the detection monochromator set to $660 \mathrm{~nm}$ (the shoulder in Figure 2) instead of $680 \mathrm{~nm}$ (the maximum in the Chl $a$ emission band), a much larger contribution of Chl $b$ was evident. All of the values for the efficiencies of energy transfer are summarized in Table 1.

Time-Resolved Fluorescence. Decay-associated fluorescence spectra for the CP26 complexes are plotted in Figure 4. The fluorescence from all of the samples is described well by three components corresponding to lifetimes of $3.2 \pm$ $0.2,5.5 \pm 0.5$, and $1.2 \pm 0.3 \mathrm{~ns}$. The first component has a maximum at $684 \mathrm{~nm}$ and corresponds to the decay of the vibrationally equilibrated lowest excited singlet state of $\mathrm{Chl}$ $a$. The second component has a maximum at $680 \mathrm{~nm}$ and represents the decay of the lowest excited singlet state of $\mathrm{Chl} b$. This component is most pronounced in the CP26 sample incorporating zeaxanthin, consistent with the fact that Gaussian deconvolution of the steady state fluorescence line shape (Figure 2) of this sample revealed $\mathrm{Chl} b$ emission. The $1.2 \pm 0.3 \mathrm{~ns}$ component is also most pronounced in the CP26 complex containing zeaxanthin compared to the other samples. It is tempting to ascribe this fast component to selective quenching of $\mathrm{Chl}$ excited singlet states by zeaxanthin. However, the component is present in all the CP26 complexes to at least some degree (Figure 4). Although the quantum yield measurements do show a small effect of the different xanthophylls on the level of fluorescence (Figure 1), most likely, the $1.2 \pm 0.3 \mathrm{~ns}$ component is due to $\mathrm{Chl} a$ molecules in a protein environment different from that occupied by the Chl $a$ molecules that decay in $3.2 \pm 0.2 \mathrm{~ns}$.
Heterogeneity of $\mathrm{Chl}$ binding sites that leads to multiple fluorescence lifetime components is not uncommon for protein complexes $(42,43)$. The fact that the $1.2 \pm 0.3 \mathrm{~ns}$ component is most pronounced in the CP26 sample containing zeaxanthin may simply be an indication that incorporation of this xanthophyll leads to more heterogeneity in that sample or to a different distribution of the population of Chls in alternate protein environments compared to the other samples. Whatever the case, the fluorescence lifetime experiments are in general agreement with the steady state fluorescence results: The control, lutein, and violaxanthin $\mathrm{CP} 26$ samples behave very similarly with respect to lifetime components and relative amplitudes. In the zeaxanthin CP26 sample, the fast, $1.2 \pm 0.3$ ns component exhibited increased amplitude while the $3.2 \pm 0.2 \mathrm{~ns}$ component exhibited decreased amplitude. These effects are very similar to those previously reported for $\mathrm{CP} 29$ (44).

\section{CONCLUSIONS}

The efficiencies of singlet energy transfer from the xanthophyll to $\mathrm{Chl}$ and the spectroscopic properties and extents of $\mathrm{Chl}$ fluorescence quenching by the xanthophylls have been measured in recombinant CP26 protein complexes containing a single xanthophyll, violaxanthin, zeaxanthin, or lutein. The steady state data demonstrate that the efficiency of light harvesting from the xanthophyll to $\mathrm{Chl} a$ is relatively high and insensitive to the particular xanthophyll that is present in the complex. A small effect of the different xanthophylls is observed on the extent of quenching of steady state Chl fluorescence, but the mechanism by which steady state fluorescence quenching is actually obtained is not yet clear. It has been proposed that fluorescence quenching may be triggered by the protonation of glutamate 166, a DCCD (dicyclohexylcarbodiimide) binding site, leading to disconnection of a specific chlorophyll and the establishment of strong xanthophyll-chlorophyll interactions (45). The modulation of the fluorescence yield may also be accomplished by variations in the occupancy of xanthophyll binding sites L1 and L2, an idea consistent with the alternative hypothesis of an allosteric effect of xanthophylls on fluorescence quenching $(4,46)$. The interaction of the xanthophylls with the Chls and/or protein may affect protein folding, leading to the modification of $\mathrm{Chl}-\mathrm{Chl}$ interactions within the protein structure as well as alterations in the steady state and dynamic spectroscopic properties and photochemical behavior. A careful kinetic analysis of the rate constants of energy transfer between the xanthophylls and $\mathrm{Chl}$ obtained from fasttransient optical spectroscopic methods would be useful in this regard.

\section{REFERENCES}

1. Demmig-Adams, B., and Adams, W. W. I. (1992) Annu. Rev. Plant Physiol. Mol. Biol. 43, 599-626.

2. Horton, P., and Boyer, J. R. (1990) in Methods in Plant Biochemistry (Harwood, J. L., and Boyer, J. R., Eds.) pp 259296, Academic Press, London.

3. Krause, G. H., and Weis, E. (1992) Annu. Rev. Plant Physiol. Mol. Biol. 42, 313-349.

4. Horton, P., Ruban, A. V., Rees, D., Pascal, A. A., Noctor, G., and Young, A. J. (1991) FEBS Lett. 292, 1-4.

5. Horton, P., and Ruban, A. V. (1992) Photosynth. Res. 34, 375385 . 
6. Ruban, A. V., Rees, D., Noctor, G. D., Young, A., and Horton, P. (1991) Biochim. Biophys. Acta 1059, 355-360.

7. Ruban, A. V., Rees, D., Pascal, A. A., and Horton, P. (1992) Biochim. Biophys. Acta 1102, 39-44.

8. Ruban, A. V., Young, A. J., and Horton, P. (1993) Plant Physiol. 102, 741-750.

9. Ruban, A. V., Young, A. J., and Horton, P. (1994) Biochim. Biophys. Acta 1186, 123-127.

10. Ruban, A. V., Young, A. J., Pascal, A. A., and Horton, P. (1994) Plant Physiol. 104, 227-234.

11. Krieger, A., Moya, I., and Weis, E. (1992) Biochim. Biophys. Acta 1102, 167-176.

12. Briantais, J.-M., Vernotte, C., Picaud, M., and Krause, G. H. (1979) Biochim. Biophys. Acta 548, 128-138.

13. Demmig-Adams, B. (1990) Biochim. Biophys. Acta 1020, $1-24$.

14. Demmig-Adams, B., and Adams, W. W., III (1993) in Carotenoids in Photosynthesis (Young, A. J., and Britton, G., Eds.) pp 206-251, Chapman and Hall, London.

15. Demmig-Adams, B., and Adams, W. W. (1996) Trends Plant Sci. 1, 21-26.

16. DeCoster, B., Christensen, R. L., Gebhard, R., Lugtenburg, J., Farhoosh, R., and Frank, H. A. (1992) Biochim. Biophys. Acta 1102, 107-114.

17. Frank, H. A., Cua, A., Chynwat, V., Young, A., Gosztola, D., and Wasielewski, M. R. (1994) Photosynth. Res. 41, 389395.

18. Owens, T. G. (1994) in Photoinhibition of Photosynthesis (Baker, N. R., and Bowyer, J. R., Eds.) pp 95-107, Bios Scientific Publishers, Oxford, U.K.

19. Frank, H. A., Desamero, R. Z. B., Chynwat, V., Gebhard, R., van der Hoef, I., Jansen, F. J., Lugtenburg, J., Gosztola, D., and Wasielewski, M. R. (1997) J. Phys. Chem. A 101, 149157.

20. Polivka, T., Herek, J. L., Zigmantas, D., Akerlund, H. E., and Sundstrom, V. (1999) Proc. Natl. Acad. Sci. U.S.A. 96, 49144917.

21. Frank, H. A., Bautista, J. A., Josue, J. S., and Young, A. J. (2000) Biochemistry 39, 2831-2837.

22. Peter, G. F., and Thornber, J. P. (1991) J. Biol. Chem. 266, $16745-16754$.

23. Jansson, S. (1994) Biochim. Biophys. Acta 1184, 1-19.

24. Bassi, R., Pineau, B., Dainese, P., and Marquardt, J. (1993) Eur. J. Biochem. 212, 297-303.

25. Sandonà, D., Croce, R., Pagano, A., Crimi, M., and Bassi, R. (1998) Biochim. Biophys. Acta 1365, 207-214.

26. Bassi, R., Sandonà, D., and Croce, R. (1997) Physiol. Plant. $100,769-779$.
27. Walters, R. G., and Horton, P. (1995) in Photosynthesis: From Light to Biosphere (Mathis, P., Ed.) pp 299-302, Kluwer Academic Publishers, Dordrecht, The Netherlands.

28. Walters, R. G., Ruban, A. V., and Horton, P. (1997) Proc. Natl. Acad. Sci. U.S.A. 93, 14204-14209.

29. Pesaresi, P., Sandonà, D., Giuffra, E., and Bassi, R. (1997) FEBS Lett. 402, 151-156.

30. Bergantino, E., Dainese, P., Cerovic, Z., Sechi, S., and Bassi, R. (1995) J. Biol. Chem. 270, 8474-8481.

31. Giuffra, E., Zucchelli, G., Sandonà, D., Croice, R., Cugini, D., Garlaschi, F. M., Bassi, R., and Jennings, R. C. (1997) Biochemistry 36, 12984-12993.

32. Giuffra, E., Cugini, D., Croce, R., and Bassi, R. (1996) Eur. J. Biochem. 238, 112-120.

33. Ros, F., Bassi, R., and Paulsen, H. (1998) Eur. J. Biochem. 253, 653-658.

34. Melhuish, W. H. (1962) J. Opt. Soc. Am. 52, 1256-1258.

35. Vasil'ev, S., and Bruce, D. (1998) Biochemistry 37, 1104611051.

36. Origin(1999) Microcal Software, Inc., Northampton, MA.

37. Kühlbrandt, W., Wang, D. N., and Fujiyoshi, Y. (1994) Nature 367, 614-621.

38. Bassi, R., Croce, R., Cugini, D., and Sandonà, D. (1999) Proc. Natl. Acad. Sci. U.S.A. 96, 10056-10061.

39. Croce, R., Remelli, R., Varotto, C., Breton, J., and Bassi, R. (1999) FEBS Lett. 456, 1-6.

40. Andersson, P. O., Gillbro, T., Ferguson, L., and Cogdell, R. J. (1991) Photochem. Photobiol. 54, 353-360.

41. Frank, H. A., Bautista, J. A., Josue, J., Pendon, Z., Hiller, R. G., Sharples, F. P., Gosztola, D., and Wasielewski, M. R. (2000) J. Phys. Chem. B 104, 4569-4577.

42. Connelly, J. P., Müller, M. G., Hucke, M., Gatzen, G., Mullineaux, C. W., Ruban, A. V., Horton, P., and Holzwarth, A. R. (1997) J. Phys. Chem. B 101, 1902-1909.

43. Hodges, M., and Moya, I. (1988) Biochim. Biophys. Acta 935, 41-52.

44. Crimi, M., Dorra, D., Bosinger, C. S., Giuffra, E., Bassi, R., and Holzwarth, A. R. (1998) in Proceedings of the International Congress on Photosynthesis (Garab, G., Ed.) pp 333336, Budapest, Hungary.

45. Crofts, A. R., and Yerkes, C. T. (1994) FEBS Lett. 352, 265270.

46. Ruban, A. V., Phillip, D., Young, A. J., and Horton, P. (1998) Photochem. Photobiol. 68, 829-834.

BI001160Q 